\title{
Drug interactions and risk of acute bleeding leading to hospitalisation or death in patients with chronic atrial fibrillation treated with warfarin
}

\author{
Christiane Gasse', Jennifer Hollowell ${ }^{2}$, Christoph R. Meier ${ }^{3}$, Walter E. Haefeli' \\ 'Department of Internal Medicine VI, Clinical Pharmacology and Pharmacoepidemiology, University of Heidelberg, Germany \\ ${ }^{2}$ AstraZeneca R\&D, Mölndal, Sweden \\ ${ }^{3}$ Basel Pharmacoepidemiology Unit, Division of Clinical Pharmacology \&Toxicology, University of Basel, Switzerland
}

\begin{abstract}
Summary
Although drug interactions with warfarin are an important cause of excessive anticoagulation, their impact on the risk of serious bleeding is unknown. We therefore performed a cohort study and a nested case-control analysis to determine the risk of serious bleeding in 4152 patients (aged 40-84 years) with nonvalvular atrial fibrillation (AF) taking long-term warfarin $(>3$ months). The study population was drawn from the UK General Practice Research Database. More than half (58\%) of eligible patients used potentially interacting drugs during continuous warfarin treatment. Among 45 identified cases of incident idiopathic bleeds (resulting in hospitalisation within 30 days or death within 7 days) and 143 matched controls, more cases than controls took $\geq 1$ potentially interacting drug within the preceding
\end{abstract}

30 days $(62.2 \%$ vs. $35.7 \%$ ) and used $>4$ drugs (polypharmacy) within the preceding 90 days ( $80.0 \%$ vs. $66.4 \%$ ). Conditional logistic regression analysis yielded an odds ratio (OR) of $3.4(95 \%$ confidence interval [Cl]: I.4-8.5) for the risk of serious bleeding in patients treated with warfarin and $\geq I$ drugs potentially increasing the effect of warfarin vs. warfarin alone adjusted for polypharmacy, diabetes, hypertension, heart failure, and thyroid disease; the adjusted OR for the combined use of warfarin and aspirin vs. warfarin alone was $4.5(95 \% \mathrm{Cl}$ : I.I-I8.I). We conclude that concurrent use of potentially interacting drugs with warfarin is associated with a 3 to 4.5 -fold increased risk of serious bleeding in long-term warfarin users.

\section{Keywords}

Atrial fibrillation, bleeding, drug interactions, warfarin

Thromb Haemost 2005; 94: 537-43

\section{Introduction}

Warfarin has been shown in clinical trials to be effective for stroke prophylaxis in patients with atrial fibrillation (AF) (1). In clinical practice, however, achieving effective and safe anticoagulation with warfarin is difficult because the anticoagulant effect can be influenced by many intrinsic and extrinsic factors (2-5). In particular, drug interactions with warfarin are a major cause of excessive anticoagulation (2-5) and hence could be an important cause of bleeding in patients taking warfarin. Use of warfarin in patients with chronic non-valvular AF is of particular concern since these patients are typically elderly with multiple co-morbidities requiring concomitant drug therapies increasing the risk for drug-drug interactions.
Most documented interactions with warfarin are based on small case series or single cases, or have been extrapolated from in-vitro or animal studies (6). A few epidemiological studies have estimated the risk of bleeding during concurrent use of oral anticoagulants and other specific drugs, including paracetamol, aspirin and other platelet-inhibiting drugs, or NSAIDS (7-10), but a systematic investigation is lacking.

The objective of the present study, therefore, was to investigate the association between warfarin and the concurrent use of potentially interacting drugs and the risk of serious bleeding in patients receiving warfarin for prevention of AF-related stroke. 


\section{Materials and methods}

\section{Study design}

We conducted a longitudinal follow-up study of patients with AF treated with warfarin to estimate the effect of concomitant use of potentially interacting drugs on the incidence of serious bleeding (resulting in hospitalisation or death), with a nested case-control analysis to further quantify the risks adjusted for potential confounders.

\section{Study population}

The study population was drawn from the General Practice Research Database (GPRD), a computerised database of longitudinal patient records collected from a panel of general practitioners (GPs) in the United Kingdom. Participating GPs use computers in their offices to record patient medical information such as demographics, 'significant' medical diagnoses, outpatient visits, hospitalisations and prescribed drugs. The validity of the database with regard to quality and completeness of the data has been extensively documented (11).

The study cohort consisted of patients with AF (aged 40-84 years), permanently registered with one of the participating practices during the study period (January 1991 to April

Table I: List of potentially interacting drugs.

\begin{tabular}{|c|c|}
\hline Allopurinol & Ketoconazole \\
\hline Amiodarone & Ketorolac \\
\hline Aspirin & Levothyroxine Sodium \\
\hline Azapropazone & Liothyronine Sodium \\
\hline Aztreonam & Lymecycline \\
\hline Benorilate & Mefenamic acid \\
\hline Bezafibrate & Meloxicam \\
\hline Bicalutamide & Methylphenidate \\
\hline Cefaclor & Metronidazole \\
\hline Cefamandole & Miconazole \\
\hline Cefazolin & Minocycline \\
\hline Cefixime & Moxolactam \\
\hline Cefmetazole & Nalidixic acid \\
\hline Cefotetan & Norfloxacin \\
\hline Ceftriaxone & Ofloxacin \\
\hline Celecoxib & Omeprazole \\
\hline Chloramphenicol & Oxymetholone \\
\hline Chlortetracycline & Oxytetracycline \\
\hline Cimetidine & Paracetamol \\
\hline Ciprofibrate & Paroxetine \\
\hline Ciprofloxacin & Phenylbutazone \\
\hline Citalopram & Piroxicam \\
\hline Clarithromycin & Proguanil \\
\hline Clofibrate & Propafenone \\
\hline Clopidogrel & Rofecoxib \\
\hline Danazol & Sertraline \\
\hline Dextropropoxyphene & Simvastatin \\
\hline Diclofenac & Stanozolol \\
\hline Diflunisal & Sulfadiazine \\
\hline Dipyridamole & Sulfadoxine \\
\hline Disulfiram & Sulfamethoxazol \\
\hline Doxycycline & Sulfinpyrazone \\
\hline Erythromycin & Sulfinpyrazone \\
\hline Fenofibrate, & Sulfisoxazole \\
\hline Fluconazole & Sulindac \\
\hline Fluorouracil & Tamoxifen \\
\hline Fluoxetine & Testosterone \\
\hline Flurbiprofen & Tetracycline \\
\hline Flutamide & Ticlopidine \\
\hline Fluvoxamine & Toremifene \\
\hline Gemfibrozil & Trimethoprim \\
\hline Ibuprofen & Valproate \\
\hline Ifosfamide & Venlafaxine \\
\hline Itraconazole & Zafirlukast \\
\hline
\end{tabular}

2001), who had a first ever warfarin prescription for AF during the study period and continued treatment for more than 90 days. Patients were excluded if they had an increased risk of bleeding due to (a) pre-existing conditions (prior coagulation disorders, cancer, peptic ulcer disease, alcohol or drug abuse), (b) history of major bleeding prior to starting warfarin treatment, or (c) high-intensity warfarin therapy associated with a prosthetic heart valve.

\section{Follow-up}

Patients in this cohort were followed from day 90 of warfarin treatment (Start date) until the earliest of (End date): warfarin discontinuation or break in warfarin exposure, occurrence of an incident bleed of any severity, the development of an exclusion diagnosis (coagulation disorder etc.), pregnancy, age 85 years, death or end of study period.

\section{Warfarin exposure}

We could not directly estimate the duration of warfarin treatment from the number of tablets prescribed because warfarin dosages are generally not fixed, and dosage instructions are most commonly recorded in the computer records as "as directed". Periods of current warfarin exposure were therefore determined by assuming an exposure duration of 90 days per prescription, i.e. warfarin exposure was deemed to be continuous provided that the interval between prescriptions did not exceed 90 days. In the event of more than 90 days between prescriptions, current exposure was deemed to end 90 days after the last prescription. We followed patients only during the first period of continuous warfarin exposure, starting on day 90 of treatment and ending on the last day of follow-up or the last day of continuous treatment. The first 90 days of treatment were excluded from our analysis because higher rates of bleeding are generally observed during this period due to pre-existing lesions and INR fluctuations during treatment initiation (12).

\section{Concomitant exposure to potentially interacting drugs} We selected the British National Formulary as a reference for potentially interacting drugs that may increase the effects of warfarin or with antiplatelet effect because it is an important, unbiased source of reference information for GPs in the UK and is the source of information on potential drug interactions referenced in the British Society for Haematology guidelines on oral anticoagulation $(13,14)$. Of the many documented potential drug interactions with warfarin, the drugs we studied were only those for which appropriate, clinically relevant information was available during the study period $(2,6,15,16)$ to increase the specificity of our analysis. The list of drugs is, additionally, very similar to the interacting drugs mentioned in the recently published American College of Chest Physician guidelines on antithrombotic therapy (17). We defined interacting drugs as those listed as "enhancing or possibly enhancing the effect of warfarin" or "increasing the risk of bleeding due to antiplatelet effect" in combination with this agent (13). These included analgesics, antibacterial drugs, antifungals, antiplatelet drugs, hormone preparations, anti-lipidaemic drugs, non-steroidal anti-inflammatory drugs (NSAIDs), selective serotonin reuptake inhibitors (SSRIs), ulcer-healing drugs, allopurinol, and amiodarone (Table 1). Only 
oral formulations of these agents were considered; the exception was miconazole, for which oral gels and intravaginal preparations were considered because case reports indicate that miconazole is sufficiently systemically absorbed from such preparations to interact with other drugs (6). We did not include penicillins in our study because these agents are not established as increasing the anticoagulant effect of warfarin (13). There is some evidence that amoxicillin may be associated with an increased risk of over-anticoagulation, but it is unclear whether the underlying febrile disease or amoxicillin may cause an excessive warfarin response $(3,18,19)$. For the case-control analysis, interacting drugs were further subdivided into two categories: (a) drugs that may increase the warfarin effect as measured by the INR, and (b) agents that may inhibit haemostasis (i.e. aspirin, clopidogrel, dipyridamole, and ticlopidine).

Duration of exposure to potentially interacting drugs was based on the actual prescription duration, which was estimated by dividing the number of tablets by the prescribed daily dose. We estimated the total duration of potentially interacting drug exposure during current continuous warfarin exposure starting with the first potentially interacting drug taken after January 1, 1991.

\section{Case definition, ascertainment and validation}

Patients who experienced incident idiopathic bleeds during continuous warfarin exposure that resulted in hospitalisation within 30 days or death within 7 days following the bleeding event were defined as cases. Bleeds were considered non-idiopathic if they were post-surgical, due to trauma or due to a coagulation disorder or another clinical condition (e.g. infection at the site of the bleed).

All incident bleeds occurring during the follow-up period were identified and reviewed by two independent reviewers, blinded to the patient's drug exposure status, to identify potential (hospitalised/fatal) cases. To ensure complete ascertainment of haemorrhagic strokes, all strokes and cerebrovascular accidents not specified as ischaemic were additionally identified and reviewed, as were the records of all patients who died during follow-up where the cause of death was unknown, non-specific and/ or consistent with bleeding as a contributory factor (e.g. sudden death, pneumonia, septicaemia, cerebrovascular disease, anaemia). For all potential cases identified where the patient was still alive, a questionnaire was sent to the patient's GP to ascertain whether the patient had been hospitalised as a result of the bleed and to establish any known contributory causes other than warfarin. We additionally obtained copies of hospital letters from the GP and requested information from the GP about the INR at the time of the bleed whereby this was not generally available in the GPs' records. Where the event was fatal, we obtained death certificates.

\section{Cohort and case-control analyses}

We calculated the incidence of serious (hospitalised/fatal) bleeds by dividing the number of incident bleeding events during current continuous warfarin exposure by the number of patientyears of exposure. We also calculated incidence rates for serious bleeds during current warfarin exposure with and without concurrent use of potentially interacting drugs.
For the case-control analysis, cases were matched with up to 6 warfarin-exposed controls on the basis of age ( \pm 3 years if no exact match), gender, practice and index date, where the index date was the date of each case's bleeding event. Cases and controls were defined as exposed to a potential warfarin interaction if an interacting drug has been prescribed within 30 days prior to the bleeding event (cases) or index date (controls). By definition, both cases and controls were exposed to warfarin on the date of prescription of the potentially interacting drug. Polypharmacy was defined as treatment with more than 4 prescription drugs including warfarin in the 30 days preceding the index date.

We used conditional logistic regression to estimate odds ratios and their $95 \%$ confidence intervals (CI) for the risk of serious bleeding associated with exposure to potentially interacting drugs in combination with warfarin compared with exposure to warfarin alone, adjusted for diabetes, hypertension, heart failure, thyroid disease, and polypharmacy. All analyses were performed using STATA 7.0 (Statistics/Data Analysis, College Station, Texas, USA).

\section{Results}

We identified 4152 eligible patients with AF commencing longterm warfarin therapy during the study period. Fifty eight percent of patients were male and $57 \%$ were aged 70 years or older. Female patients tended to be older; $69 \%$ of female patients were older than 70 years compared with $49 \%$ of male patients.

\section{Cohort analysis}

We observed a total of 3740.8 patient-years of continuous warfarin exposure among the 4152 patients in the study cohort; these patients received an average of 11.6 (median 4) warfarin prescriptions during follow-up before a break in treatment occurred. Thirty-three percent of patients received warfarin continuously for more than a year. This group contributed 1587.4 patient-years of continuous warfarin exposure, i.e. $43.5 \%$ of all observed continuous exposure time. More than half (58\%) of the 4152 patients were exposed to potentially interacting drugs at some time during current continuous warfarin exposure and, overall, patients were exposed to potentially interacting drugs more than one third (37\%) of the time (Table 2).

Some 2283 patients were censored before the end of the study period due to the end of continuous warfarin treatment without developing a bleeding event or another exclusion criterion while 1869 patients were continuously treated until they were censored from the cohort for other reasons: 432 developed a bleed of any severity, 187 were censored due to an exclusion criterion, 1 became pregnant, 33 reached the age of 85,133 died and 1083 reached the end of the study period.

Of the 432 observed bleeding events and 133 patients who died during follow-up, we identified a total of 340 incident idiopathic bleeds of any severity, of which 294 were of minor or moderate severity and 46 were classified as cases ( 7 fatal; 39 hospitalised non-fatal). Five of the cases involved intracranial bleeding (3 fatal, 2 hospitalised non-fatal), 15 of the bleeds were gastro-intestinal (3 fatal, 12 hospitalised non-fatal), 13 of the cases experienced epistaxis (all hospitalised non-fatal), and the re- 
Table 2: Characteristics of the study population and observed exposure time to warfarin (Patients years at risk (PYAR)) in all patients and in patients with use of potentially interacting drugs during warfarin exposure.

\begin{tabular}{|c|c|c|c|c|}
\hline & \multicolumn{2}{|l|}{ All patients } & \multicolumn{2}{|c|}{$\begin{array}{l}\text { Patients with interacting drug } \\
\text { use during warfarin exposure }\end{array}$} \\
\hline & No. of patients (\%) & PYAR & No. of patients (\%) ${ }^{b}$ & PYAR \\
\hline $\begin{array}{l}\text { Total (from } 90 \text { days } \\
\text { after first warfarin pre- } \\
\text { scription) }\end{array}$ & $4152(100)$ & 3740.8 & $2407(58.0)$ & 1373.3 \\
\hline \multicolumn{5}{|l|}{ Gender } \\
\hline Male & $2409(58.0)$ & 2209.4 & $1372(57.0)$ & 758.4 \\
\hline Female & $1743(42.0)$ & 1531.4 & $1035(59.4)$ & 614.9 \\
\hline \multicolumn{5}{|c|}{ Age (at first warfarin prescription) } \\
\hline $40-49$ & $127(3.1)$ & 91 & $68(53.5)$ & 34 \\
\hline $50-59$ & $467(I I .2)$ & 322.3 & $235(50.3)$ & 121.3 \\
\hline $60-69$ & $1179(28.4)$ & 1075.7 & $724(61.4)$ & 415.5 \\
\hline $70-79$ & $1898(45.7)$ & 1766.2 & $1118(58.9)$ & 626.5 \\
\hline 80-84 & $481(11.6)$ & 485.6 & $262(54.5)$ & 176 \\
\hline
\end{tabular}

maining 13 bleeds occurred at various sites, including one fatal pleural haemorrhage.

Overall, the crude incidence of serious bleeding (fatal and non-fatal) during current continuous warfarin exposure was 1.2 per 100 patient-years at risk (PYAR). The rate varied according to whether or not the patient was concomitantly exposed to potentially interacting drugs. During warfarin exposure alone the rate was 0.9 serious bleeds per 100 PYAR, increasing to 1.8 serious bleeds per 100 PYAR during periods of concomitant exposure to potentially interacting drugs (incidence rate ratio: 2.05 [95\% CI: 1.1-3.9]).

Of the 88 potentially interacting drugs considered in this analysis, 56 were taken concomitantly by warfarin users. Eight of these drugs were involved in 25 non-fatal and in 3 fatal bleeding events (2 GI-bleeds, 1 pleural haemorrhage) (Table 3). Bleeding sites among patients who used warfarin in combination with aspirin were epistaxis, purpura, haematemesis and melaena, none of them was fatal.
The highest incidence rates, although only based on one case each, were found for miconazole and metronidazole. In both patients, the potentially interacting drug was newly added. In the first patient, miconazole was administered as an oral gel for the treatment of mouth sores; the patient was admitted with spontaneous bleeding and a prothrombin index of $>5 \mathrm{~min} 17$ days after miconazole had been added. The patient was also a long-term user of paracetamol. The second patient was admitted with haemorrhage and an INR of 4.5 two days after oral metronidazole was started for the treatment of a skin infection. We did not find evidence of bleeding events associated with any other antibacterials. Sulfamethoxazole was only prescribed to 8 patients (0.1 PYAR), trimethoprim to 209 patients (6 PYAR), macrolides to 194 patients (5.5 PYAR), and quinolones to 104 patients (2.6 PYAR) during continuous warfarin use.

Dosage information was assessed for paracetamol and aspirin use. The average daily documented dose for paracetamol among cases varied from $885 \mathrm{mg}$ to $2900 \mathrm{mg}$, and had been prescribed for at least 4 weeks prior to their bleeding events. Doses for aspirin varied from $75 \mathrm{mg}$ to $325 \mathrm{mg}$ /day.

Exposure to non-aspirin NSAIDS was low in this population (100.9 PYAR all combined) and none of the non-aspirin NSAIDS was involved in a bleeding event.

We obtained information on INRs at the time of the bleed in 7 cases: Excessive anticoagulation was present in the two patients on miconazole and metronidazole, see above; five cases had INRs within the therapeutic range for stroke prophylaxis (2.0-3.0) of which 2 were not exposed to potentially interacting drugs and 3 were exposed to paracetamol $(n=2)$ and allopurinol $(n=1)$.

\section{Nested case-control analysis}

The case-control analysis was based on 45 cases and 143 matched controls (no matched controls could be found for the remaining case, a 70-year-old man who experienced epistaxis while exposed to warfarin but not taking any drugs known to interact with this agent), all of whom were exposed (by definition) to current continuous warfarin at the index date. The characteristics of cases and controls are shown in Table 4. The distribution of sex differs between cases and controls because of unequal numbers of obtainable matched controls per case. Twenty-eight

Table 3: Incidence rates of serious bleeding events during concomitant use of warfarin and interacting drugs.

\begin{tabular}{|c|c|c|c|c|}
\hline Drug & $\begin{array}{l}\text { No. of patients with a bleeding } \\
\text { event leading to hospitalisation } \\
\text { or death }\end{array}$ & $\begin{array}{l}\text { No. of patients with at least } \\
\text { one prescription during current } \\
\text { warfarin exposure }\end{array}$ & $\begin{array}{l}\text { PYAR during current } \\
\text { warfarin exposure }\end{array}$ & $\begin{array}{c}\text { Incidence rate } \\
\text { (cases/l00 PYAR) }\end{array}$ \\
\hline Allopurinol & 5 & 180 & 148.7 & 3.4 \\
\hline Amiodarone & $5^{b}$ & 643 & $4 \mid 7.1$ & 1.2 \\
\hline Aspirin & 5 & 450 & 205.0 & 2.4 \\
\hline Levothyroxine & 2 & 234 & 215.3 & 0.9 \\
\hline Metronidazole & 1 & 38 & 2.6 & 38.5 \\
\hline Miconazole & I & 16 & 2.4 & 41.7 \\
\hline Omeprazole & 2 & 146 & 62.1 & 3.2 \\
\hline Paracetamol & $10^{b}$ & 1149 & 262.2 & 3.8 \\
\hline Paracetamol + Dextropropoxyphene & $4^{b}$ & 397 & 96.5 & 4.1 \\
\hline
\end{tabular}


Table 4: Characteristics of cases and controls and risk of bleeding.

\begin{tabular}{|c|c|c|c|c|}
\hline & $\begin{array}{l}\text { Cases } \\
(n=45)\end{array}$ & $\begin{array}{l}\text { Controls } \\
(n=143)\end{array}$ & $P$ value & $\begin{array}{l}\text { Odds ratio } \\
(95 \% \mathrm{Cl})^{\mathrm{a}}\end{array}$ \\
\hline \multicolumn{5}{|l|}{ Gender, $\mathbf{N}$} \\
\hline Male & $28(62.2 \%)$ & $84(58.7 \%)$ & & \\
\hline Female & $17(37.8 \%)$ & $59(41.3 \%)$ & & \\
\hline \multicolumn{5}{|l|}{ Age, $\mathrm{N}$} \\
\hline $40-49$ & 0 & 2 & & \\
\hline $50-59$ & 2 & 7 & & \\
\hline $60-69$ & 20 & 48 & & \\
\hline $70-79$ & 22 & 81 & & \\
\hline $80-84$ & 1 & 5 & & \\
\hline \multicolumn{5}{|l|}{ Concomitant disease, $\mathbf{N}$} \\
\hline Diabetes & $6(13.3 \%)$ & $12(8.4 \%)$ & 0.33 & $1.7(0.5-6 . .8)$ \\
\hline Heart failure & $26(57.8 \%)$ & $59(41.3 \%)$ & 0.05 & $1.5(0.7-3.2)$ \\
\hline Hypertension & $19(42.2 \%)$ & $62(43.4 \%)$ & 0.89 & $0.9(0.4-1.9)$ \\
\hline Thyroid disease & $5(11.1 \%)$ & $16(11.2 \%)$ & 0.99 & $0.9(0.3-3.4)$ \\
\hline \multicolumn{5}{|c|}{ No. of drugs received within 30 days prior to the index date, $N$} \\
\hline $\mathrm{I}$ & I $(2.2 \%)$ & $3(2.1 \%)$ & & \\
\hline $2-4$ & $8(17.8 \%)$ & $45(31.5 \%)$ & & \\
\hline$>4$ (Polypharmacy) & $36(80.0 \%)$ & $95(66.4 \%)$ & 0.08 & $1.2(0.4-3.4)$ \\
\hline $\begin{array}{l}\text { Use of drugs increasing } \\
\text { the effect of warfarin } \\
\text { within } 30 \text { days prior to } \\
\text { the bleeding event, } N\end{array}$ & $25(55.6 \%)$ & $48(33.6 \%)$ & 0.008 & $3.4(1.4-8.5)$ \\
\hline $\begin{array}{l}\text { Use of aspirin in } \\
\text { combination with warfarin } \\
\text { within } 30 \text { days prior to } \\
\text { the bleeding event, } \mathbf{N}\end{array}$ & $5(11.1 \%)$ & $5(3.5 \%)$ & 0.05 & $4.5(1.1-18.1)$ \\
\hline
\end{tabular}

aDerived using a conditional logistic regression model adjusting for diabetes, hypertension, heart failure, thyroid disease, and polypharmacy. Cl: confidence interval.
Table 5: Interacting drugs prescribed to cases and controls within $\mathbf{3 0}$ days prior to the index date during current continuous exposure to warfarin.

\begin{tabular}{|c|c|c|}
\hline Drug name & $\begin{array}{l}\text { Cases exposed to } \\
\text { interacting drugs } \\
(n=28)^{a}\end{array}$ & $\begin{array}{l}\text { Controls exposed to } \\
\text { interacting drugs } \\
(n=5 I)^{b}\end{array}$ \\
\hline Allopurinol & $5(17.9 \%)$ & $5(9.8 \%)$ \\
\hline Amiodarone & $5(17.9 \%)$ & $8(15.7 \%)$ \\
\hline Apazone & 0 & I (2.0\%) \\
\hline Aspirin & $5(17.9 \%)$ & $5(9.8 \%)$ \\
\hline Ciprofloxacin & 0 & $2(3.9 \%)$ \\
\hline Diclofenac & 0 & $2(3.9 \%)$ \\
\hline Erythromycin & 0 & I ( $2.0 \%)$ \\
\hline Fluoxetine & 0 & I (2.0\%) \\
\hline Levothyroxine & $2(7.1 \%)$ & $7(13.7 \%)$ \\
\hline Mefenamic acid & 0 & I (2.0\%) \\
\hline Metronidazole & I (3.6\%) & 0 \\
\hline Miconazole & I (3.6\%) & 0 \\
\hline Omeprazole & $2(7.1 \%)$ & $2(3.9 \%)$ \\
\hline Oxytetracycline & 0 & $\mathrm{I}(2.0 \%)$ \\
\hline Paracetamol & $10(35.7 \%)$ & $16(31.4 \%)$ \\
\hline $\begin{array}{l}\text { Paracetamol + } \\
\text { Dextropropoxyphene }\end{array}$ & $4(14.3 \%)$ & $8(15.7 \%)$ \\
\hline Piroxicam & 0 & I (2.0\%) \\
\hline Simvastatin & 0 & $3(5.9 \%)$ \\
\hline
\end{tabular}

paracetamol (all preparations combined) (cases vs controls: $31.1 \%$ [95\% CI: $18.1-46.6 \%$ ] vs $16.8 \%$ [95\% CI: $11.1-23.9 \%]$ ), followed by allopurinol (cases vs controls: $11.1 \%$ [95\% CI: $3.7-24.1 \%$ ] vs $3.5 \%$ [95\% CI: $1.1-5.0 \%]$ ] and amiodarone (cases vs controls: $11.1 \%$ [95\% CI: $3.7-24.1 \%]$ vs $5.6 \%$ [95\% CI: $2.4-10.7 \%]$ ).

\section{Discussion}

cases $(62.2 \%)$ and $51(35.7 \%)$ controls were exposed to potentially interacting drugs or antiplatelet agents in combination with warfarin.

Crude odds ratios were 3.2 (95\% CI: 1.5-7.1) for the risk of serious bleeding associated with the use of drugs that may increase the effect of warfarin compared with warfarin alone, and 3.6 (95\% CI: 1.0-12.6) for the risk associated with the use of antiplatelet agents in combination with warfarin compared with warfarin alone. After adjusting for co-morbidities and polypharmacy, the adjusted odds ratios were $3.4(95 \%$ CI: $1.4-8.5)$ and 4.5 (95\% CI: 1.1-18.1), respectively (Table 4).

Table 5 shows the potentially interacting drugs prescribed to cases and controls during the 30 days prior to the index date. Six cases were concomitantly exposed to more than one potentially interacting drug in combination with warfarin; three patients took paracetamol in addition to levothyroxine, omeprazole, and miconazole respectively, two patients were exposed to aspirin in addition to allopurinol and amiodarone respectively, and one patient was exposed to allopurinol and amiodarone. The most frequently prescribed potentially interacting drug among cases was
In this observational study of UK patients receiving long-term therapy with warfarin for prevention of AF-related stroke, we found that potentially interacting drugs were used in combination with warfarin for more than one third of the time and that more than half of the patients were exposed to a potential interaction during the study period. We found a 3 -fold increased risk of bleeding leading to hospitalisation or death in warfarin users concomitantly taking potentially interacting drugs, and a 4.5 -fold increased risk for patients using aspirin in combination with warfarin.

More than half of the patients $(61 \%)$ with a serious bleeding event in our study were exposed to potentially interacting drugs or an antiplatelet agent in combination with warfarin before the bleeding event. This proportion is comparable with the results of a recent (uncontrolled) trend study in the US in hospitalized patients with warfarin-associated haemorrhage where $62 \%$ used drugs known to potentiate the bleeding risk before the bleeding event (20). All fatal cases in that study were taking potentially potentiating drugs compared with 3 out of 7 fatal bleeding cases 
in our study. Case fatality may vary between different clinical settings or countries depending on anticoagulation monitoring and the management of the drug-drug interactions and over-anticoagulation.

The rate of serious bleeding observed in our study is lower than that reported in a previous GPRD study (1.2 vs. 3.1 bleeds per 100 PYAR in patients with AF) (21).The rates, however, are not directly comparable since in the present study we deliberately excluded patients with pre-existing conditions associated with an increased risk of bleeding (e.g. cancer, peptic ulcer disease), and we additionally excluded the initiation phase of treatment when the risk of bleeding is highest (12). A lower rate of bleeding is therefore unsurprising in our cohort at a lower underlying risk of bleeding than the study population of Hollowell et al. (2003).

These restrictions may also explain the differing result of a recently published study that did not find any significant association between drug interactions and major bleeding (OR 1.33; 95\% CI 0.96-.86) (10). The study included patients with other major bleeding risk factors and patients who had just started treatment $(40 \%)$ and were therefore at an increased risk of bleeding irrespective of potential drug interactions (10). Moreover, drug-drug interactions are a sub-group of adverse drug reactions which are predictable and therefore preventable in most instances (22). Thus the strength of the association between potentially interacting drugs and adverse outcomes (the drug interaction) strongly depends on the awareness and management of potentially interacting drugs by treating physicians.

Our study confirms the results of two recent studies that reported that concurrent antiplatelet therapy was independently associated with an increased risk of major bleeding $(10,23)$. However, our estimate is higher with wide confidence intervals.

Of the 88 interacting drugs included in our analysis only 8 were involved in serious bleeding events in the present study (11). Although our study was not designed to evaluate the risks associated with individual drugs, the estimated crude incidence rates suggest a possible increased risk of bleeding associated with the use of warfarin in combination with paracetamol (alone or in combination with other analgesics), allopurinol, metronidazole, miconazole, omeprazole, or aspirin. However, 6 of the cases were exposed to more than one potentially interacting drug and since we analysed each drug separately, our methods will have overestimated the risks associated with some of these drugs. While allopurinol, metronidazole and miconazole have previously been reported to cause clinical relevant drug interactions $(2,6,24,25)$, our findings add to the evidence that paracetamol may increase the risk of bleeding among warfarin users. Most patients were 'heavy' paracetamol users taking dosages above the Hylek threshold of $9.8 \mathrm{~g}$ per week and had been prescribed paracetamol for at least 4 weeks prior to their bleeding events (5). It has been suspected that the association between paracetamol use and increased risk of bleeding may be due to confounding by indication (7); paracetamol is generally considered safer than aspirin in combination with warfarin because it does not affect platelets or cause gastric bleeding. In the present study, we excluded patients who might have avoided aspirin due to the risk of GI bleeding (i.e. those with pre-existing pepticulcer disease, history of GI bleeding or alcohol abuse), so we consider this unlikely to explain the association between paracetamol use and increased risk of bleeding.

It is worth noting that the warfarin-miconazole interaction can occur with oral gel formulations of miconazole $(6,26)$. Physicians should therefore closely monitor the patient's INR for an adequate period even if miconazole is given as an oral gel formulation.

The pattern of interacting drugs among cases in our study differed from the one reported by Kucher et al. where the most commonly used drugs were quinolone antibiotics $(32 \%)$, levothyroxine (15\%), simvastatin (10\%), and amiodarone (10\%) (20). Patterns of interacting drugs may vary in different clinical settings or countries because of differences in the use of drugs and knowledge about potential drug interactions.

Our study has some limitations: firstly, we may underestimate the risk of bleeding episodes due to drug interactions with warfarin because we had no information on over-the-counter drugs (in particular paracetamol, aspirin, or other NSAIDs) and herbal or nutritional products, which can also interact with warfarin (24). Furthermore, our definition of exposure to potentially interacting drugs did not differentiate between newly started and long-term therapies, nor did we consider the impact of changes in dosage. Since changes in drug use may pose a greater risk of over-anticoagulation, this may have diluted the observed effect. Many of the bleeds involving allopurinol, amiodarone, and paracetamol, however, occurred during chronic use. For allopurinol and amiodarone, an effect on prothrombin time has been observed when the drugs are started whereas the effect of paracetamol seems to be dose-dependent and related to the duration of exposure (6). While it would have been possible to refine our analysis to focus on periods of exposure immediately following therapy changes, the appropriate exposure time windows for such an analysis are unclear, particularly in the case of amiodarone and paracetamol. A second limitation is the lack of INR data. It has recently been reported that drug interactions with warfarin may lead to over-anticoagulation (27) and that overanticoagulation increases the risk of bleeding 3 -fold in patients with atrial fibrillation (28) and up to 6-fold in an unrestricted patient population with different indications for warfarin treatment (29). Unfortunately INR data were generally not available for analysis in the present study because INR values are typically documented in patient booklets rather than in the GP office computer in cases where the patient attends a hospital clinic for INR monitoring. We were only able to obtain information on INRs at the time of the bleed in 7 patients, five of whom had INRs within the therapeutic range for stroke prophylaxis $(2.0-3.0)$. Though the risk of over-anticoagulation may be prevented by increasing the frequency of monitoring (and, in turn, warfarin dosage adjustment) when patients are exposed to interacting drugs, we had no means of assessing whether the treating physicians actively managed any potential drug interactions in the latter patients by increasing the frequency of INR monitoring and warfarin dosage adjustment. Moreover, drug interactions with warfarin do not necessarily result in INR changes. While inhibitors of warfarin metabolism, e.g. amiodarone, or drugs that change the kinetics of clotting factors, e.g. levothyroxine, may increase INR levels (pharmacokinetic effects) additive effects of warfarin in combination with a drug with platelet inhibiting properties may not be 
detected by INR monitoring (pharmacodynamic effects). Indeed, a recent study found that serial INRs are poor predictors of haemorrhagic events in patients receiving long-term anticoagulation treatment and reported the majority of bleeding events with INRs in the therapeutic range (30). Consequently studies that investigated the association of oral anticoagulants and interacting drugs and over-anticoagulation (INR $>6.0$ ) as a proxy for bleeding risk might have underestimated the total bleeding risk of the combined use of warfarin and interacting drugs.

In conclusion, our study demonstrates that drug interactions are an independent risk factor for serious bleeding in patients on long-term warfarin therapy for stroke prophylaxis, and that levels of usage of such potentially interacting drugs are relatively high. A need therefore exists not only to increase awareness among physicians on how best to minimise the risks associated with the use of interacting drugs, but also for practical guidance regarding the timing, frequency and duration of additional monitoring required when specific drugs are used in conjunction with warfarin.

\section{Acknowledgements}

The authors would like to thank all participating general practitioners for their cooperation and help in the conduct of this study. We thank Joachim Troost and Verena Schneider for participation in the case review process. We also thank the Boston Collaborative Drug Surveilance Program (BCDSP) for providing and managing the data and Steve Winter and Simon Sharp for editorial assistance. Editorial assistance was funded by AstraZeneca.

\section{References}

1. Risk factors for stroke and efficacy of antithrombotic therapy in atrial fibrillation. Analysis of pooled data from five randomized controlled trials. Arch Intern Med 1994; 154: 1449-57.

2. Wells PS, Holbrook AM, Crowther NR et al. Interactions of warfarin with drugs and food. Ann Intern Med 1994; 121: 676-83.

3. Panneerselvam S, Baglin C, Lefort $\mathrm{W}$ et al. Analysis of risk factors for over-anticoagulation in patients receiving long-term warfarin. $\mathrm{Br} \mathrm{J}$ Haematol 1998; 103: 422-4.

4. Brigden ML, Kay C, Le A et al. Audit of the frequency and clinical response to excessive oral anticoagulation in an out-patient population. Am J Hematol 1998; 59: 22-7.

5. Hylek EM, Heiman H, Skates SJ et al. Acetaminophen and other risk factors for excessive warfarin anticoagulation. JAMA 1998; 279: 657-62.

6. Stockley IH. Stockley's Drug Interactions. Sixth edition. London, Pharmaceutical Press; 2002.

7. Johnsen SP, Sorensen HT, Mellemkjoer L et al. Hospitalisation for upper gastrointestinal bleeding associated with use of oral anticoagulants. Thromb Haemost 2001; 86: 563-8.

8. Landefeld CS, Beyth RJ. Anticoagulant-related bleeding: clinical epidemiology, prediction, and prevention. Am J Med 1993; 95: 315-28.

9. Clarke MF, Boardman HS. Interaction between warfarin and oral terbinafine. Systematic review of interaction profile of warfarin is needed. BMJ 1998; 317 : 205-6.

10. Shireman TI, Howard PA, Kresowik TF et al. Combined anticoagulant-antiplatelet use and major bleeding events in elderly atrial fibrillation patients. Stroke 2004; 35: 2362-7.

11. Jick SS, Kaye JA, Vasilakis-Scaramozza C et al. Validity of the general practice research database. Pharmacotherapy $2003 ; 23$ : 686-9.
12. Steffensen FH, Kristensen K, Ejlersen E et al Major haemorrhagic complications during oral anticoagulant therapy in a Danish population-based cohort. J Intern Med 1997; 242: 497-503.

13. British National Formulary, Edition 41. London, Pharmaceutical Press; 2001.

14. Guidelines on oral anticoagulation: third edition Br J Haematol 1998; 101: 374-87.

15. Demirkan K, Stephens MA, Newman KP et al. Response to warfarin and other oral anticoagulants: effects of disease state. South Med J 2000; 93: 448-54. 16. Hirsh J, Dalen JE, Anderson DR et al. Oral anticoagulants: mechanism of action, clinical effectiveness, and optimal therapeutic range. Chest 2001; 119 S8-S21.

17. Ansell J, Hirsh J, Poller L et al. The pharmacology and management of the vitamin $\mathrm{K}$ antagonists: the Seventh ACCP Conference on Antithrombotic and Thrombolytic Therapy. Chest 2004; 126 (3 Suppl): S204-S33.

18. Harder S, Thürmann P. Clinically important drug interactions with anticoagulants. An update. Clin Pharmacokinet 1996; 30: 416-44.

19. Visser LE, Penning-van Beest FJA, Kasbergen AAH et al. Overanticoagulation associated with combined use of antibacterial drugs and acenocoumarol or phenprocoumon anticoagulants. Thromb Haemost 2002; 88: 705-10.

20. Kucher N, Castellanos LR, Quiroz R et al. Time trends in warfarin-associated hemorrhage. Am J Cardiol 2004; 94: 403-6.

21. Hollowell J, Ruigomez A, Johansson S et al. The incidence of bleeding complications associated with warfarin treatment in general practice in the United Kingdom. Br J Gen Pract 2003; 53: 312-4.

22. Bates DW, Cullen DJ, Laird N et al. Incidence of adverse drug events and potential adverse drug events.Im- plications for prevention. ADE Prevention Study Group. JAMA 1995; 274: 29-34.

23. Buresly K, Eisenberg MJ, Zhang X et al. Bleeding complications associated with combinations of aspirin, thienopyridine derivatives, and warfarin in elderly patients following acute myocardial infarction. Arch Intern Med 2005; 165: 784-9.

24. Hansten P, Wittkowsky AK. Warfarin drug interactions. In: Ansell JE, Oertel LB, Wittkowsky AK (eds). Managing Oral Anticoagulation Therapy: Clinical and Operational Guidelines. Wolters Kluwer Health, Inc., St Louis (MO); 2004.

25. Klasco RK (ed). DRUGDEX ${ }^{\circledR}$ System, 1st ed. Thomson MICROMEDEX ${ }^{\circledR}$, Greenwood Village (CO); 2004

26. Visser LE, Penning-van Beest FJA, Kasbergen $\mathrm{AAH}$ et al. Overanticoagulation associated with combined use of antifungal agents and coumarin anticoagulants. Clin Pharmacol Ther 2002; 71: 496-502.

27. Howard PA, Ellerbeck EF, Engelman KK et al. The nature and frequency of potential warfarin drug interactions that increase the risk of bleeding in patients with atrial fibrillation. Pharmacoepidemiol Drug Saf 2002; 11: 569-76.

28. Reynolds MW, Fahrbach K, Hauch O et al. Warfarin anticoagulation and outcomes in patients with atrial fibrillation: a systematic review and metaanalysis. Chest 2004; 126: 1938-45.

29. Koo S, Kucher N, Nguyen PL et al. The effect of excessive anticoagulation on mortality and morbidity in hospitalized patients with anticoagulant-related major hemorrhage. Arch Intern Med 2004; 164: 1557-60.

30. Kucher N, Conolly S, Beckman JA et al. International normalized ratio increase before warfarin-associated hemorrhage: brief and subtle. Arch Intern Med 2004; 164: 2176-9. 